\title{
ChemComm
}

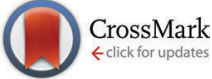

Cite this: Chem. Commun., 2015, 51, 8679

Received 2nd March 2015 Accepted 16th April 2015

DOI: $10.1039 / c 5 c c 01791 b$

www.rsc.org/chemcomm

\section{A flow cytometer-based whole cell screening toolbox for directed hydrolase evolution through fluorescent hydrogels $\uparrow$}

\author{
Nina Lülsdorf, $\ddagger^{\mathrm{a}}$ Christian Pitzler, $\ddagger^{\mathrm{b}}$ Michael Biggel, ${ }^{\mathrm{b}}$ Ronny Martinez, ${ }^{\mathrm{c}}$ \\ Ljubica Vojcic ${ }^{b}$ and Ulrich Schwaneberg ${ }^{\star a b}$
}

\begin{abstract}
A high throughput whole cell flow cytometer screening toolbox was developed and validated by identifying improved variants (1.3-7-fold) for three hydrolases (esterase, lipase, cellulase). The screening principle is based on coupled enzymatic reaction using glucose derivatives which yield upon hydrolysis a fluorescent-hydrogel-layer on the surface of E. coli cells.
\end{abstract}

Flow cytometer-based screening systems are, despite their extremely high throughput (up to $1.8 \times 10^{7}$ events per hour) and a competitively high sensitivity, rarely used in directed evolution campaigns. This can be attributed to challenges in the compartmentalization technology such as double emulsion formation, dispersity of the emulsion, substrate and product compatibility, and stability of compartments. ${ }^{1-3}$ Flow cytometry whole cell based screening systems for directed evolution require fluorogenic substrates which can diffuse inside the cell and remain entrapped upon conversion into fluorescent products. ${ }^{4-7}$ The latter has been reported in directed evolution campaigns for three specific enzymes (P450 monooxygenase, $\mathrm{N}$-acetylgalactosaminidase, protease).$^{5-7}$ Therefore, novel screening principles which are generally applicable are of high interest for protein engineering and biocatalysis. Recently, we reported a proof of concept for a novel screening principle named Fur-Shell which is based on fluorescent hydrogel formation around E. coli cells with a phytase as an example. In the Fur-Shell screening platform, a whole cell acts as a compartment in which $\mathrm{H}_{2} \mathrm{O}_{2}$ is formed through a coupled reaction of phytase and glucose oxidase; subsequently a fluorescent monomer is co-polymerized in a fluorescent polyester hydrogel shell formed around $E$. coli cells expressing active phytase variants. ${ }^{8}$ Significantly, the proof of

\footnotetext{
${ }^{a}$ DWI-Leibniz Institut für Interaktive Materialien, Forckenbeckstraße 50, 52056 Aachen, Germany

${ }^{b}$ Lehrstuhl für Biotechnologie, RWTH Aachen University, Worringerweg 3,

52074 Aachen, Germany. E-mail: u.schwaneberg@biotec.rwth-aachen.de;

Fax: +4924180 22387; Tel: +492418024170

${ }^{c}$ EW-Nutrition GmbH, Nattermannallee 1, 50829 Köln, Germany

$\dagger$ Electronic supplementary information (ESI) available: Material and methods, additional results. See DOI: 10.1039/c5cc01791b

‡ Joint first authors.
}

concept of the Fur-Shell technology overcomes technical limitations in flow cytometry-based screening systems in terms of compartmentalization and leakage of a fluorogenic substrate and/or fluorescent product. In the current report we advanced the screening principles of the Fur-Shell technology into a general high throughput screening toolbox for directed evolution of hydrolases by establishing and validating screening protocols for three hydrolases: a $p$-nitrobenzyl esterase from Bacillus licheniformis ( $p$ NBEBL $),{ }^{9}$ a Bacillus subtilis lipase A (BSLA), ${ }^{10}$ and a cellulase (CelA2) isolated from a metagenome library by Streit et al. ${ }^{11}$ Validation was performed for each of the three hydrolases through a single round of directed evolution by screening an epPCR random mutagenesis library. All three hydrolases address enzymes which are of significant synthetic and/or industrial importance (e.g. esterases and lipases are used in laundry detergents, in the synthesis of pharmaceuticals, and in food processing; ${ }^{12}$ cellulases are applied in e.g. depolymerisation of cellulose and in food industry ${ }^{13-15}$ ). Fig. 1 shows the principle of the Fur-Shell technology in four steps. In step 1 the gene diversity library is generated by epPCR. Subsequently, insert and vector fragments are cloned by PLICing and the mutant library is transformed and expressed in E. coli cells. ${ }^{16}$ Step 2 comprises the Fur-Shell technology, which relies on the conversion of a substrate $(\beta-\mathrm{D}-(+)$-glucose pentaacetate for esterase and lipase; cellobiose for cellulase) into $\beta$-D-glucose. Subsequently, in a glucose oxidase coupled reaction $\mathrm{H}_{2} \mathrm{O}_{2}$ and glucono- $\delta$-lactone are produced. Through Fenton reaction, radical species from $\mathrm{H}_{2} \mathrm{O}_{2}$ are generated, initiating a PEG based co-polymerization of the fluorescent Polyfluor 570 monomer. ${ }^{8}$ E. coli cells expressing active enzyme variants are surrounded by a fluorescent hydrogel shell and can in step 3 be analysed and sorted by flow cytometer at rates of around 5000 events per second. The sorted $E$. coli cells expressing active hydrolase variants are plated on agar plates and afterwards transferred for screening in microtiter plates (MTPs) (step 4a). Alternatively, as reported here in step $4 \mathrm{~b}$, performance parameters such as low cell survival of $E$. coli cells $(<8 \%)$ were optionally addressed by introducing a plasmid isolation step of sorted cells to rescue 


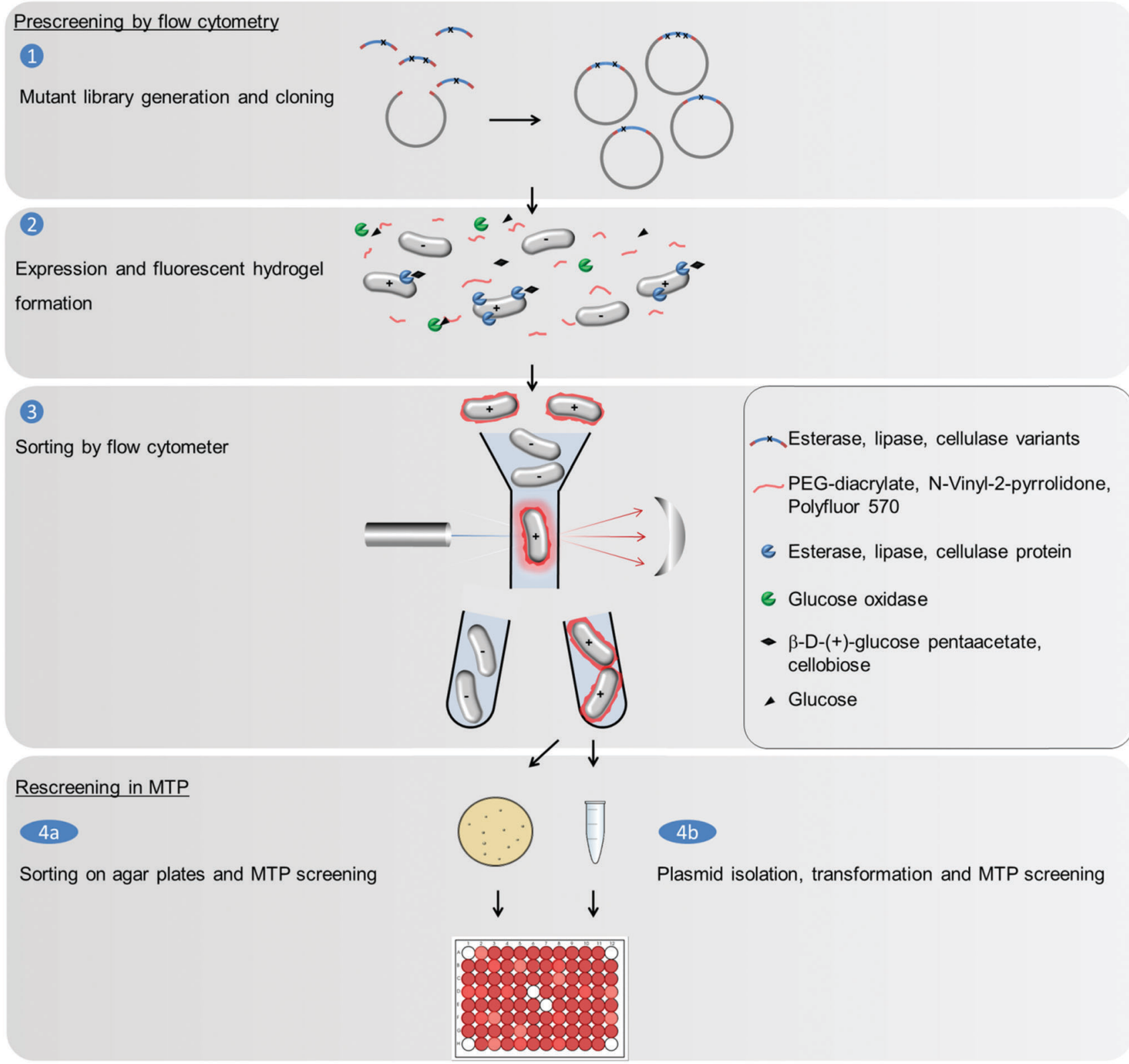

Fig. 1 Flow cytometer-based sorting principle of the Fur-Shell toolbox for hydrolases in four steps. Step 1: library generation by epPCR and subsequent cloning by PLICing, step 2: E. coli BL21-gold (DE3) cells producing enzyme variants are incubated with substrate, glucose oxidase and fluorescent labelled monomers (Polyfluor 570). Fluorescent hydrogel is formed around cells expressing active enzyme variants (+) which allows in step 3 analysis and sorting (enrichment) by flow cytometer at a rate of about 5000 events per second. Finally the E. coli BL21-gold (DE3) cells are either plated on LB agar plates (step 4a) or used for plasmid isolation and transformation (step 4b), in both cases followed by transferring clones into MTPs.

mutated genes that encoded improved hydrolase variants in non-viable $E$. coli cells. ${ }^{8}$ Isolated plasmids are subsequently transformed into competent $E$. coli BL21-gold (DE3) cells and most beneficial variants were identified after screening of hydrolase clones (at least 360 clones per hydrolase) in 96-well MTP format. Confocal microscopy images showing an overlay of transmission and fluorescence were recorded in order to show the difference in the fluorescent signal of $E$. coli BL21gold (DE3) cells producing either active hydrolases (pET22b(+)$p$ NBEBL, BSLA, or CelA2) or cells harbouring pET22b(+) (Fig. 2) (see ESI $\dagger$ ). For $E$. coli BL21-gold (DE3) cells expressing active hydrolase variants (Fig. 2D: esterase $p$ NBEBL; Fig. 2E: lipase
BSLA; Fig. 2F: cellulase CelA2) a strong fluorescent signal was detected which confirms hydrogel formation through incorporation of Polyfluor 570. E. coli BL21-gold (DE3) cells harbouring pET22b(+) (Fig. 2A-C) showed little to no fluorescence. In Fig. 3 flow cytometry analysis of Fur-Shell labelled E. coli BL21-gold (DE3) cells are shown and confirm the visual impression of recorded confocal microscopy images. Comparison of Fig. 3(A, D; B, E; C, F) shows a significant difference in fluorescence intensity among $E$. coli cells expressing active hydrolases and those harbouring an empty vector. The fluorescent intensity of $E$. coli cells harbouring an empty vector was around 20-fold lower compared to E. coli cells expressing wildtype hydrolases (Fig. 3D: esterase $p$ NBEBL; Fig. 3E: lipase 


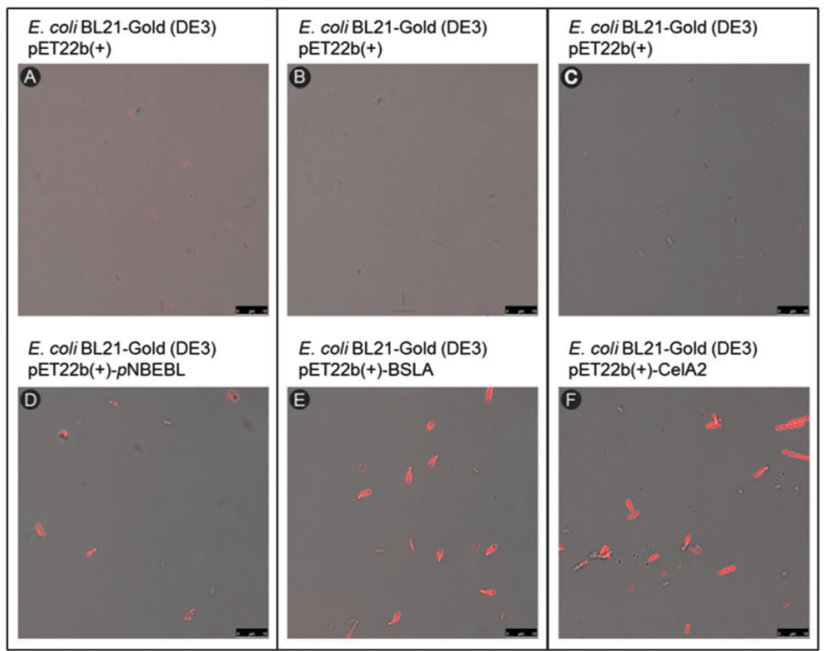

Fig. 2 Overlay of fluorescence and transmission images which were recorded by confocal microscope analysis (see ESI $\dagger$ ), showing the empty vector signal of $E$. coli $\mathrm{BL} 21$-gold (DE3) $\mathrm{pET} 22 \mathrm{~b}(+)$ incubated with the substrate $\beta$-D-(+)-glucose pentaacetate (A, B) or with the substrate cellobiose (C). D, E: E. coli BL21-gold (DE3) pET22b(+)-pNBEBL/E. coli BL21-gold (DE3) $\mathrm{pET} 22 \mathrm{~b}(+)$-BSLA were incubated with the substrate $\beta$-D-(+)-glucose pentaacetate. F: E. coli BL21-gold (DE3) pET22b(+)-CelA2 was incubated with the substrate cellobiose.
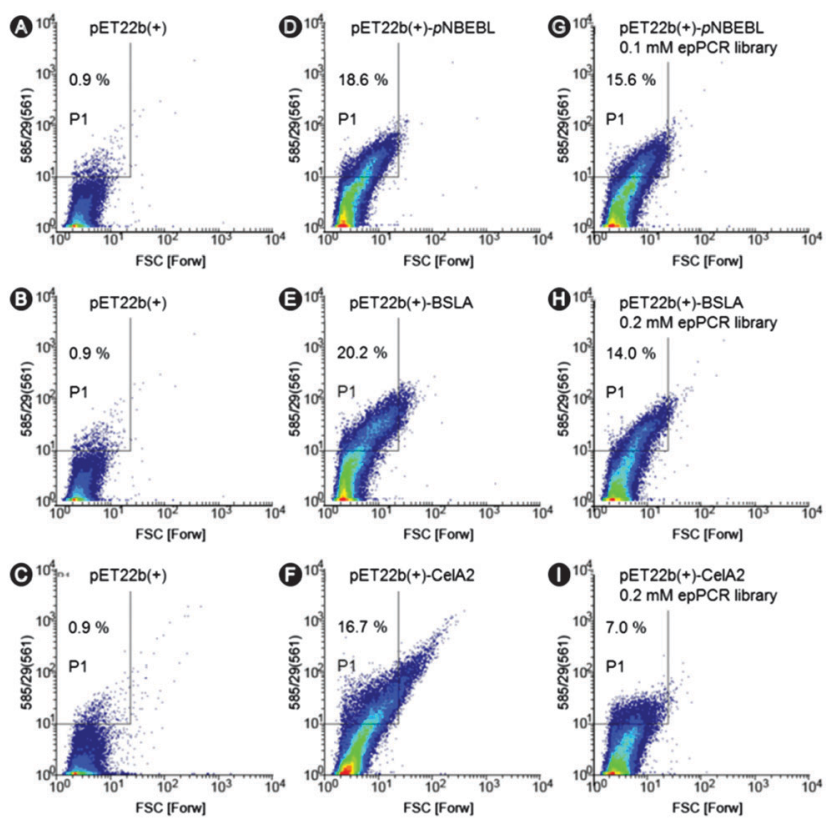

Fig. 3 Flow cytometry analysis of Fur-Shell labelled E. coli cells. Density plots were used to indicate the fluorescent signals generated through $E$. coli cells coated with fluorescent hydrogel shells (recorded by forward scatter (FSC) versus fluorescence signal $\left(\lambda_{\mathrm{ex}} 561 \mathrm{~nm} / \lambda_{\mathrm{em}} 585 \mathrm{~nm}\right)$ ). Gate (P1) was set to sort all events with a size $<3 \mu \mathrm{m}$ in order to exclude associated cells. ${ }^{17} \mathrm{E}$. coli BL21-gold (DE3) cells were incubated with $\beta-D-(+)$ glucose pentaacetate $(A, B$, $D, E, G, H$ ) or cellobiose (C, F, I) as substrates. A-C: E. coli BL21-gold (DE3) $\mathrm{pET} 22 \mathrm{~b}(+)$ cells were used as a negative control to determine the threshold for fluorescence. D-F: E. coli BL21-gold (DE3) pET22b(+)-pNBEBL, -BSLA, -CelA2 wildtype cells were used as a positive control. Random mutagenesis libraries were generated with epPCR: $0.1 \mathrm{mM} \mathrm{MnCl}$; $p \mathrm{NBEBL}$ (G) $0.2 \mathrm{mM} \mathrm{MnCl}_{2}$ for $\operatorname{BSLA}(\mathrm{H})$, and $0.2 \mathrm{mM} \mathrm{MnCl} 2$ for CelA2 (I).
BSLA; Fig. 3F: cellulase CelA2). One epPCR random mutagenesis library per hydrolase gene (esterase pnbebl, lipase bsla and cellulase cela2) was generated using 0.1 or $0.2 \mathrm{mM} \mathrm{MnCl}_{2}$ with an average mutation frequency of 2.2 (pnbebl, $0.1 \mathrm{mM} \mathrm{MnCl}_{2}$ ), 13.2 (bsla, $0.2 \mathrm{mM} \mathrm{MnCl}_{2}$ ), and 4.2 (cela2, $0.2 \mathrm{mM} \mathrm{MnCl}_{2}$ ) mutations per $\mathrm{kb}$ (see $\mathrm{ESI} \dagger$ ). The populations of all three mutant libraries were analysed by flow cytometer and showed a reduced fluorescent signal due to the presence of inactive hydrolase variants (Fig. 3G: esterase $p$ NBEBL, Fig. 3H: lipase BSLA, Fig. 3I: cellulase CelA2). The sorting gate $\mathrm{P} 1$ was set to minimize background fluorescent signal (0.9\%, see Fig. 3A-C) from E. coli BL21-gold (DE3) cells harbouring an empty vector ( $Y$-axis). This means $>99 \%$ of the negative, non-fluorescent population is excluded in the sorting gate and in return solely positive, active cells are sorted. Additionally, P1 was adjusted using calibration beads with the forward scatter laser ( $X$-axis) to contain only single cells being smaller than $3 \mu \mathrm{m}$ in size in order to exclude associated cells. ${ }^{17}$ E. coli BL21-gold (DE3) cells expressing active hydrolase variants were sorted and collected (Fig. 1, step 3). DNA of $5 \times 10^{5}$ sorted $E$. coli cells was recovered by using option $4 \mathrm{~b}$ (Fig. 1, step 4b: plasmid isolation to maintain diversity and to compensate for the survival rate $(7 \%)) .{ }^{8}$ Subsequently, isolated plasmids were transformed for expression into E. coli BL21-gold (DE3) cells and plated on LB-agar (see ESI $\dagger$ ). Variants of the three hydrolases (in total 2160 clones) were transferred into 96-well MTPs for subsequent screening. In case of $p$ NBEBL the identical substrate $(\beta-\mathrm{D}-(+)$-glucose pentaacetate) was employed for flow cytometry and MTP screening (see principle and procedure in $\mathrm{ESI}_{\dagger}^{\dagger}$ ); in case of CelA2 the similar substrate 4-methylumbelliferyl$\beta$-D-cellobioside (4-MUC) was employed, and in case of the BSLA $p$-nitrophenyl acetate ( $p$ NPA) was used due to better sensitivity and established screening procedures in 96-well MTP format. ${ }^{18,19}$ Enrichment factors were determined by activity measurements and calculated by dividing the percentage of active populations after and before flow cytometry sorting (number of clones analysed before sorting: 180 variants per hydrolase; number of clones analysed after flow cytometry sorting: 360 variants ( $p$ NBEBL) or 900 variants per hydrolase (BSLA; CelA2)). In detail, enrichment was efficient for the CelA2 population (11.7-fold enrichment, $38 \%$ active population in sorted libraries); in case of $p$ NBEBL (1.3-fold enrichment, $41 \%$ active population in sorted libraries) and BSLA (1.5-fold enrichment, $44 \%$ active population in sorted libraries) low enrichment was obtained which was unexpected due to the adjustment of the P1 sorting gate. A decrease reaction time for hydrogel formation from $10 \mathrm{~min}$ to $2 \mathrm{~min}$, as well as decrease in monomer concentration did not yield a further improvement in enrichment factors. Nevertheless, in all cases a sufficiently high number of active hydrolase variants was obtained for 96-well MTP screening. Two improved variants out of rescreening of 8 variants for $p$ NBEBL, 14 variants for BSLA and 8 variants for CelA2 in MTP format were kinetically characterized in detail and compared to the corresponding wildtypes (Fig. S1, ESI $\dagger$ and Table 1). The esterase $p$ NBEBL variant E1 (E256G, G401V) showed an impressive 7-fold higher $k_{\text {cat }}$ and 2-fold lower $K_{\mathrm{M}}$ value compared to wildtype $p$ NBEBL, indicating a high specific activity. In E1 (E256G, G401V), the E256G substitution has not been reported yet and is located on 
Table 1 Kinetic characterization of $p N B E B L, B S L A$ and CelA2 was performed regarding $K_{\mathrm{M}}, K_{\text {cat }}$ and $U \mathrm{mg}^{-1}$. pNBEBL was kinetically characterized at $22^{\circ} \mathrm{C}$ with the fluorometric Amplite screening system (see Fig. S2A, ESI). BSLA was kinetically characterized with the colourimetric substrate $p N P A$ (at $22{ }^{\circ} \mathrm{C}, \mathrm{pH}$ 6.5) (see Fig. S2B, ESI). CelA2 was kinetically characterized with the fluorogenic substrate 4-MUC (at $30^{\circ} \mathrm{C} ; \mathrm{pH}$ 7.2) (see Fig. S2C, ESI). Units are defined as: $1 \mathrm{U}$ of $p$ NBEBL catalyzes the conversion of $1 \mathrm{mmol} \beta-\mathrm{D}-(+)$-glucose pentaacetate per second, $1 \mathrm{U}$ of BSLA catalyzes the conversion to $1 \mu \mathrm{mol} p N \mathrm{~N}$ per minute, $1 \mathrm{U}$ of CelA2 catalyzes the conversion to $1 \mu \mathrm{mol} 4-\mathrm{MU}$ per minute

\begin{tabular}{lccl}
\hline Enzyme (substitution) & $K_{\mathrm{M}}[\mu \mathrm{M}]$ & $k_{\mathrm{cat}}\left[\mathrm{l} \mathrm{s}^{-1}\right]$ & $\begin{array}{l}\mathrm{U} \mathrm{g}^{-1} \\
{\left[\mathrm{mmol} \mathrm{s}^{-1} \mathrm{~g}^{-1}\right]}\end{array}$ \\
\hline$p$ NBEBL wildtype & $540 \pm 170$ & $26 \pm 1$ & $481 \pm 24$ \\
E1 (E256G, G401V) & $270 \pm 50$ & $186 \pm 4$ & $3415 \pm 68$ \\
E2 (F313S) & $300 \pm 100$ & $169 \pm 7$ & $3106 \pm 107$ \\
\hline & & & $\mathrm{U} \mathrm{mg}^{-1}$ \\
Enzyme (substitution) & $K_{\mathrm{M}}[\mu \mathrm{M}]$ & $k_{\mathrm{cat}}\left[1 \mathrm{~s} \mathrm{~s}^{-1}\right]$ & {$\left[\mu \mathrm{mol} \mathrm{min}^{-1} \mathrm{mg}^{-1}\right]$} \\
\hline BSLA wildtype & $1259 \pm 92$ & $3.4 \pm 0.1$ & $10.7 \pm 0.3$ \\
L1 (Y139D) & $1893 \pm 241$ & $4.3 \pm 0.2$ & $13.4 \pm 0.7$ \\
L2 (R60Q, S166G) & $1807 \pm 243$ & $4.2 \pm 0.2$ & $13.1 \pm 0.7$ \\
\hline & & & $\mathrm{U} \mathrm{mg}$ \\
Enzyme (substitution) & $K_{\mathrm{M}}[\mu \mathrm{M}]$ & $k_{\mathrm{cat}}\left[1 \mathrm{~s} \mathrm{~s}^{-1}\right]$ & {$\left[\mu \mathrm{mol} \mathrm{min}^{-1} \mathrm{mg}^{-1}\right]$} \\
\hline CelA2 wildtype & $170 \pm 8$ & $0.15 \pm 0.02$ & $0.14 \pm 0.02$ \\
C1(V37A, E275G, E398V) & $189 \pm 9$ & $0.26 \pm 0.03$ & $0.22 \pm 0.03$ \\
C2 (N135S) & $172 \pm 9$ & $0.26 \pm 0.03$ & $0.23 \pm 0.03$ \\
\hline
\end{tabular}

surface of $p$ NBEBL. The G401V substitution is located next to the $\mathrm{H} 400$ which is part of the catalytic triad, and the position was reported to increase thermostability of $p$-nitrobenzyl esterase. ${ }^{20}$ The BSLA variant L1 (Y139D) showed 1.3-fold increase in $k_{\text {cat }}$ compared to the wildtype BSLA. Amino acid substitution Y139D is located in close vicinity to the substrate binding pocket and the side chain is turned to the outside of the molecule. Position Y139 in BSLA has not been reported yet. CelA2 variant C1 (V37A, E275G, E398V) showed a 1.9-fold increase in $k_{\text {cat }}$ compared to wildtype CelA2. Position E275 is located on the surface between two $\beta$-sheets, position E398 is located in the middle of a $\alpha$-helix, and position V37 was not covered by the homology model which was generated on the basis of a glycosyl hydrolase family 9 (GH9) cellobiosidase from Clostridium cellulovorans (PBD ID: 3RX7). None of the three substituted positions in CelA2 variant $\mathrm{C} 1$ have been reported yet. Compared to standard directed enzyme evolution campaigns (low mutagenesis frequency, 1200-15000 variants screened per round; 1.5-2.5-fold improved activity ${ }^{21}$ ) the obtained improvements were comparable to one round of evolution in case of BSLA and CelA2 and impressive in case of $p$ NBEBL (7-fold). The number of clones screened in 96-well MTPs (on average 720) is significantly lower compared to standard directed evolution experiments. Therefore the Fur-Shell technology is from our point of view best used as prescreening system in order to isolate active cells from large cell populations ( $>10^{7}$ cells) into a MTP format and thereby to minimize screening efforts in a cost effective manner. The three hydrolase examples show that we advanced the Fur-Shell screening principle into a general platform for directed hydrolase evolution by reporting first validated protocols for an esterase, a lipase, and a cellulase. The developed protocols are easy in use and time-efficient when compared to other reported flow cytometry-based screening systems in directed evolution. The challenges in emulsion compartmentalization based systems such as leakage of fluorogenic substrates and/or fluorescent products and crosstalk of double emulsions are solved in Fur-Shell screening platform. The principle of fluorescent hydrogel formation around whole cells can likely be expanded to alternative fluorescent hydrogels (Fur-Shells) as well as enzyme classes and has from our point of view the potential to establish flow cytometry as standard screening format in directed enzyme evolution.

Authors thank Prof. Dr Wolfgang Streit for providing the CelA2, Prof. Dr Karl-Erich Jaeger for providing BSLA, and Henkel AG \& Co. KGaA for providing the $p$ NBEBL amino acid sequence. Work was financially supported by the German Federal Ministry of Education and Research (BMBF) [FKZ: 031A165] [FKZ: 031A227F] and the Alliance "FuPol" (Funktionalisierung von Polymeren).

\section{Notes and references}

1 S. van der Graaf, C. G. P. H. Schroen and R. M. Boom, J. Membr. Sci., 2005, 251, 7-15.

2 K. Pays, J. Giermanska-Kahn, B. Pouligny, J. Bibette and F. L. Calderon, J. Controlled Release, 2002, 79, 193-205.

3 A. B. Theberge, F. Courtois, Y. Schaerli, M. Fischlechner, C. Abell, F. Hollfelder and W. T. Huck, Angew. Chem., Int. Ed., 2010, 49, $5846-5868$.

4 A. J. Ruff, A. Dennig, G. Wirtz, M. Blanusa and U. Schwaneberg, ACS Catal., 2012, 2, 2724-2728.

5 F. Courtois, L. F. Olguin, G. Whyte, A. B. Theberge, W. T. S. Huck, F. Hollfelder and C. Abell, Anal. Chem., 2009, 81, 3008-3016.

6 K. Kalidasan, Y. Su, X. Wu and S. Q. Yao, Chem. Commun., 2013, 49, 7237-7239.

7 R. Tu, R. Martinez, R. Prodanovic, M. Klein and U. Schwaneberg, J. Biomol. Screening, 2011, 16, 285-294.

8 C. Pitzler, G. Wirtz, L. Vojcic, S. Hiltl, A. Boker, R. Martinez and U. Schwaneberg, Chem. Biol., 2014, 21, 1733-1742.

9 B. Veith, C. Herzberg, S. Steckel, J. Feesche, K. H. Maurer, P. Ehrenreich, S. Baumer, A. Henne, H. Liesegang, R. Merkl, A. Ehrenreich and G. Gottschalk, J. Mol. Microbiol. Biotechnol., 2004, 7, 204-211.

10 T. Eggert, G. van Pouderoyen, B. W. Dijkstra and K. E. Jaeger, FEBS Lett., 2001, 502, 89-92.

11 N. Ilmberger, D. Meske, J. Juergensen, M. Schulte, P. Barthen, U. Rabausch, A. Angelov, M. Mientus, W. Liebl, R. A. Schmitz and W. R. Streit, Appl. Microbiol. Biotechnol., 2012, 95, 135-146.

12 T. Eggert, G. Pencreac'h, I. Douchet, R. Verger and K. E. Jaeger, Eur. J. Biochem., 2000, 267, 6459-6469.

13 Y. Sun and J. Cheng, Bioresour. Technol., 2002, 83, 1-11.

14 S. Ito, Extremophiles, 1997, 1, 61-66.

15 M. K. Bhat, Biotechnol. Adv., 2000, 18, 355-383.

16 M. Blanusa, A. Schenk, H. Sadeghi, J. Marienhagen and U. Schwaneberg, Anal. Biochem., 2010, 406, 141-146.

17 H. E. Kubitschek, J. Bacteriol., 1990, 172, 94-101.

18 C. Lehmann, F. Sibilla, Z. Maugeri, W. R. Streit, P. D. de Maria, R. Martinez and U. Schwaneberg, Green Chem., 2012, 14, 2719-2726.

19 K. Shirai, R. L. Jackson and D. M. Quinn, J. Biol. Chem., 1982, 257, 10200-10203.

20 B. Spiller, A. Gershenson, F. H. Arnold and R. C. Stevens, Proc. Natl. Acad. Sci. U. S. A., 1999, 96, 12305-12310.

21 R. Martinez, F. Jakob, R. Tu, P. Siegert, K. H. Maurer and U. Schwaneberg, Biotechnol. Bioeng., 2013, 110, 711-720. 